\title{
IDOMAL: the malaria ontology revisited
}

\author{
Pantelis Topalis ${ }^{1}$, Elvira Mitraka², Vicky Dritsou' ${ }^{1}$ Emmanuel Dialynas ${ }^{1}$ and Christos Louis ${ }^{2,3^{*}}$
}

\begin{abstract}
Background: With about half a billion cases, of which nearly one million fatal ones, malaria constitutes one of the major infectious diseases worldwide. A recently revived effort to eliminate the disease also focuses on IT resources for its efficient control, which prominently includes the control of the mosquito vectors that transmit the Plasmodium pathogens. As part of this effort, IDOMAL has been developed and it is continually being updated.

Findings: In addition to the improvement of IDOMAL's structure and the correction of some inaccuracies, there were some major subdomain additions such as a section on natural products and remedies, and the import, from other, higher order ontologies, of several terms, which were merged with IDOMAL terms. Effort was put on rendering IDOMAL fully compatible as an extension of IDO, the Infectious Disease Ontology. The reason for the difficulties in fully reaching that target were the inherent differences between vector-borne diseases and "classical" infectious diseases, which make it necessary to specifically adjust the ontology's architecture in order to comprise vectors and their populations.

Conclusions: In addition to a higher coverage of domain-specific terms and optimizing its usage by databases and decision-support systems, the new version of IDOMAL described here allows for more cross-talk between it and other ontologies, and in particular IDO. The malaria ontology is available for downloading at the OBO Foundry (http://www.obofoundry.org/cgi-bin/detail.cgi?id=malaria_ontology) and the NCBO BioPortal (http://bioportal. bioontology.org/ontologies/1311).
\end{abstract}

Keywords: Malaria, Vector borne disease, IDO, Remedies, Ontology

\section{Findings}

\section{Background}

Although eradicated from most of the non-tropical regions of the world since decades, malaria is still being considered as one of the major scourges of mankind, affecting hundreds of millions of people in the tropical regions of the world [1]. Recent years have witnessed a revival of the idea of eradicating the disease, although this time the prevailing goal is that of elimination, rather than to completely expunge it $[2,3]$. To achieve this objective emphasis has to be put on disease control, aiming at both the disease as such (prevention, diagnosis and treatment) and, most importantly, at vector control [1]. On both fronts, different measures have to be chosen and actions such as vaccine and novel antimalarial drug

\footnotetext{
* Correspondence: louis@imbb.forth.gr

${ }^{2}$ Institute of Molecular Biology and Biotechnology, Foundation for Research and Technology-Hellas and Department of Biology, University of Crete, Heraklion, Crete, Greece

${ }^{3}$ Centre of Functional Genetics, Medical School, University of Perugia, Perugia, Italy

Full list of author information is available at the end of the article
}

development, innovative strategies for vector control and vector population monitoring, etc., have to be prominently assisted by approaches based on Information Technology (IT). It becomes clear that there is a need for new effective tools that will be able to combine different, yet related datasets covering various aspects of disease (e.g. epidemiological and entomological data, intervention efforts, etc.). These tools encompass resources such as smart databases (including decision support systems), enhanced bioinformatics software and usage of technologies such as the Internet and mobile telephony for the fast transfer of data. The latter is especially crucial, given that malaria usually strikes the world's poorest areas, in countries in which general infrastructures are often under-developed.

It is now established that ontologies help overcome several difficulties encountered in the wide usage of IT resources by achieving enhanced interoperability. This is the reason why we decided to put emphasis on the development of ontologies that cover the domains of both vector borne diseases, including malaria, and the vectors
C Biomed Central

(c) 2013 Topalis et al.; licensee BioMed Central Ltd. This is an Open Access article distributed under the terms of the Creative Commons Attribution License (http://creativecommons.org/licenses/by/2.0), which permits unrestricted use, distribution, and reproduction in any medium, provided the original work is properly cited. 
that transmit their pathogens. We have therefore developed a series of ontologies [4] that describe the anatomy of mosquitoes and ticks, mosquito insecticide resistance, as well as malaria as the first disease in this category. It was early on decided that the latter ontology, IDOMAL [5], would be built as an extension to IDO, the Infectious Disease Ontology [6]. The first version of IDOMAL was made publicly available three years ago, at a time when IDO was still at a very early stage of development. This "wrong order" obviously led to some discrepancies between the two ontologies, which would have to be reconciled if IDOMAL is to be considered a bona fide extension of IDO. It is particularly important to achieve the status as early as possible, since we are in the process of developing additional ontologies in the domain of vector borne diseases such as, for example, IDODEN, a yet unpublished ontology for Dengue fever [7]. IDODEN follows the same architecture as IDOMAL, something that proved to be extremely efficient in terms of the ontology design. We therefore decided to restructure IDOMAL at this point in order to avoid major future "repair" work on several ontologies. Here, we describe both the changes introduced in IDOMAL for this purpose, as well as several other changes that have been made.

\section{Updating IDOMAL}

All significant changes introduced to IDOMAL are indicated in Table 1. In total 222 terms were added, while another 207 changes of different nature were performed. All terms and relations that are included in the ontologies described here are in italics. The first piece of update is the conversion of IDOMAL from the OBO format to OWL. The advantages and shortcomings of the one versus the other format are not to be discussed here. Given, though, the increased spread of the OWL format among bio-ontologies we decided to proceed with the conversion, at the same time making both the OBO and the OWL formatted versions available to the community. We used the OBOtoOWL script that we previously described [8] to achieve the conversion.
The next change introduced to IDOMAL could be called technical since it concerned the editing of the ontology in order to make sure that all terms of the ontology have complete is_a paths to the root, which, now, in accordance to BFO (Basic Formal Ontology) [9-11] is entity. Therefore the term role which was one of many root-level terms in previous versions, now took its proper place and is to be found under realizable entity, which is a specifically dependent continuant, which is a dependent continuant, which is a continuant, which is an entity - the root term of BFO. Furthermore, condition, which was also a top-level term, is now a sibling of role, in accordance to the BFO 1.0 specification [9-11]. Finally, we corrected typos that were found, and added a small number of terms that had either missed our attention or their inclusion was deemed necessary given the latest developments in malaria research.

A further major addition to IDOMAL concerns the inclusion of terms from the sub-domain of natural remedies and medicinal plants. Chemotherapy of malaria has been increasingly hindered by the development of resistance of Plasmodium parasites against antimalarial drugs [12]. The search for novel antimalarials, therefore, has now also turned its attention to traditional remedies, in particular natural products derived from plants. It should be stressed that, currently, one such product and its derivatives (artemisinins), against which widespread resistance had not been developed, are now under such risk [13]. To cover the domain we used, mostly, a handbook [14] that fully covers the domain. We should underline the fact that we concentrated, obviously, on terms that described substances and procedures for which a certain degree of efficacy had been previously shown. Similar to what is true for "conventional" antimalarials, we do not consider the terms included as complete, and we are planning to perform more additions if necessary.

In addition to this important addition we also decided to perform a wide "exchange" of terms. The decision was taken based on the idea that in the now rapidly expanding field of bio-ontologies the same term is often

Table 1 List of changes and corrections introduced in IDOMAL

\begin{tabular}{lll}
\hline Update element & Action & Comments \\
\hline Non availability of IDOMAL in OWL Format & Used OBOtoOWL script to generate & Both versions publically available \\
Several terms lacked "is_a" paths to top & Completed "is_a" paths for all terms & entity is now the single root term. \\
Multiple (11) root terms & Fully adopted BFO hierarchy to top-level & Imported (merged) terms from other ontologies \\
Availability of terms in other ontologies & 146 terms added & "Missing" terms \\
Absence of terms relating to natural products/remedies & 76 terms added (included in those above) & See Table 2 for details \\
Minor problems (typos, individual terms, definitions) & Edited/ completed & \\
Discrepancies with IDO & &
\end{tabular}


defined differently, and is also linked with is_a relations to terms that are different. For example, querying the Bioportal [15] one finds that the term symptom is now described in 15 distinct ontologies. Interestingly, IDO includes symptom as a quality and has no children terms, the Ontology for General Medical Science (OGMS) [16] has entity as its parent and only lists one child (pain) while, finally, the Influenza ontology (FLU) [17] also has entity as the parent of symptom (also imported from OGMS) and has 8 children listed that, though, do not include pain. It should be noted that FLU is an extension of IDO. In IDOMAL, symptom (with a large number of children) is linked to condition of the malaria host, which is obviously a child of condition. We decided to replace all children of symptom with those listed by the Symptom Ontology (SYMP) [18], in which symptom is the root. By term replacing we actually mean the merging of the terms from SYMP to those in IDOMAL. Merging, instead of replacing leaves both IDs intact within IDOMAL, and therefore if somebody has already been using IDOMAL there will be no need to perform any changes in the software that uses the ontology. The choice of SYMP was made purely on the fact that alternatives such as OGMS do not list the terms that we needed. Finally, we should state at this point that IDO is using the symptom term imported from OGMS (see below).

\section{IDOMAL and IDO}

As mentioned earlier, due to the timing of development of the two ontologies the published version of IDOMAL has some features that make it difficult, as such, to be called an extension of IDO in the latter's present form. The example stated above, i.e. the term symptom being imported from two different ontologies, exemplifies this problem while, at the same time, it also shows that the differences are not necessarily irreconcilable: the easiest solution for this kind of discrepancy would be to simply merge the two terms. There are several more examples of how some differences may be eliminated. Table 2 lists these, showing in addition the actions taken or to be taken. For example, while antiparasitic drug is a role in IDOMAL, a term antiparasitic disposition is found in IDO, defining antiparasitic material entity as entity, which bears antiparasitic disposition (IDO contains dispositions such as antibacterial, antifungal, antiparasitic, antimicrobial, antiviral). We could easily reconcile the difference by accepting that antimalarial drug in IDOMAL is a role borne by a material entity which has antiparasitic disposition and is given to a patient to treat malaria. This would follow the example set in IDO by antiseptic role (definition: A role borne by a material entity in virtue of the fact that it has an antimicrobial disposition and is applied to an anatomical entity of a living organism). There are a few more cases of discrepancy between the two ontologies and for some of them we have decided to adopt the IDO point of view. For example, endemicity that was a disposition in IDOMAL will be changed to quality of a population, and so will be the terms holoendemicity, hypoendemicity and mesoendemicity, which are absent from IDO. We have also changed resistance from quality to disposition; although a good case was made for the fact that resistance is a disposition [19], we should nevertheless state here that resistance is in most, if not all cases a genetic phenotype. And without going into further discussions, we simply state that phenotypes are usually considered to be quality, possibly because of the fact that several of them are visible (e.g. white eyes, ectopic expression, etc.). Finally, habitat, a spatial region so far in IDOMAL, has been changed to site like in IDO. What also had to be changed to fit the present ontological representation is to define breeding site as a role carried by material entity (i.e. site).

In spite of the changes made, a series of issues remain that haven't yet been resolved. Some of them, in our opinion, are relatively secondary and they could be resolved easily. For example, terms such as treatment and refractoriness which we deem to be necessary for an ontology of vector borne diseases could be carried again by IDO, from which they were obsoleted some time ago.

The remaining open issues are due to the distinctive properties of vector borne diseases. These infections are characterized by the fact that they arise through the biological interactions between three organisms (patient/ host, vector and pathogen), rather than only two as is common in the vast majority of infectious diseases. Thus, an ontology such as IDOMAL has to capture all three organisms, as well as prominently include terms on the respective populations. For example, control of malaria, eventually leading, perhaps, to its elimination, is predominantly based on vector control. This involves measures aimed, for example, at reducing mosquito populations, possibly using genetic approaches [20] or, as may be the case in the future if planned strategies succeed, at replacing vector populations with others that will simply not be able to transmit the pathogen [21].

Both IDO and IDOMAL use the BFO [9-11] as an upper level ontology. IDO would then describe the infectious disease domain, while IDOMAL would ideally be placed below it. The current structure of IDO, though, does not allow for a full deployment of the malaria domain. IDOMAL has separated several classes of terms in three main "groups" namely the patient/host, the vector and the pathogen or, to be more precise, into six groups since populations are "treated" separately. The reason for this is obvious: many terms apply to both patient/host and vector (both being metazoan). Similarly, 
Table 2 Differences between IDO and IDOMAL and steps (to be) taken to unify the ontologies

\begin{tabular}{|c|c|}
\hline & IDO-IDOMAL “comparison” \\
\hline IDO: & symptom imported from OGMS, no childrer \\
\hline IDOMAL: & native term, 39 children \\
\hline IDO: & endemicity is quality of a population \\
\hline IDOMAL: & endemicity is disposition \\
\hline IDO: & habitat is site (material entity) \\
\hline IDOMAL: & habitat is spatial region \\
\hline IDO: & breeding site absent \\
\hline IDOMAL: & Anopheles breeding site is a role \\
\hline IDO: & $\begin{array}{l}\text { epidemiological types [of an infectious } \\
\text { disease] absent }\end{array}$ \\
\hline IDOMAL: & epidemiological types of malaria present \\
\hline IDO: & $\begin{array}{l}\text { antiparasitic material entity is material entity } \\
\text { bearing antiparasitic disposition }\end{array}$ \\
\hline
\end{tabular}

IDOMAL: antiparasitic drug is a role.

IDO: resistance is a disposition

IDOMAL: resistance is quality (of (vector population)

IDO: $\quad$ infection is a material entity

IDOMAL: infection is not present; infectious disposition is not present

IDO: vector competence is not present

IDOMAL: vector competence is present

IDO: infectious disease control is absent

IDOMAL: malaria control is present

IDO: $\quad$ No terms for decision support systems, [disease] and vector control, intervention methods

IDOMAL: Corresponding terms present

IDO: refractoriness is obsolete term

IDOMAL: refractoriness is present

IDO: $\quad$ treatment is obsolete term in IDO

IDOMAL: treatment is present

IDO: $\quad$ holoendemicity / hypoendemicity / mesoendemicity are missing

IDOMAL: terms are present

IDO: quality of infectious disease is absent

IDOMAL: quality of malaria is present

IDO: process of infectious disease is absent

IDOMAL: process of malaria is present

IDO: no differentiation between host/patient and pathogen

IDOMAL: differentiates between host/patient, vector and pathogen

\section{Action}

Imported/merged terms from

Symptom Ontology

Changed in IDOMAL, now as in IDO

Changed in IDOMAL, now as in IDO

Changed to Anopheles breeding site role born by site (material entity)

Discuss with IDO developers

Needed in IDOMAL

Issue solved in IDOMAL: antimalarial drug is a role born by a material entity which has antiparasitic disposition

Changed in IDOMAL, now as in IDO

No action needed

Discuss with IDO developers

Discuss with IDO developers

Discuss with IDO developers

Discuss with IDO developers

Discuss with IDO developers

Discuss with IDO developers

Discuss with IDO developers

Discuss with IDO developers

Discuss with IDO developers
Terms are not needed in IDOMAL

Similar for antibiotic role/ disposition, etc.

Needed in IDOMAL

Needed in IDOMAL

Needed in IDOMAL

Needed in IDOMAL

Needed in IDOMAL

Needed in IDOMAL

Needed in IDOMAL, acts as a placeholder for terms relating to malaria-specific interventions.
Needed in IDOMAL, coverage of physiological and pathophysiological processes occurring in host and/or vector and/or parasite. 
the latter separation, of course, is due to the fact that several terms are specific for populations, rather than individuals; this is especially true for vector control.

Another problem that is not yet resolved is how to list in a grouped, ontologically correct form terms such as pathogen specific form of malaria and epidemiological type of malaria. A solution could be to create sub-ontologies for each one of the different forms of malaria (i.e. for $P$. falciparum, $P$. malariae, $P$. ovale and $P$. vivax); we consider this to be impractical in several obvious aspects. For the time being we keep the problematic class quality of malaria and we'll aim at finding an appropriate way to describe these features in collaboration with the IDO consortium. The class process of malaria, thus, groups a series of physiological and pathophysiological processes occurring in the patient/ host and/or the parasite. Finally, IDOMAL has no placeholders for vector-specific processes (e.g. host seeking) or qualities (e.g. vector competence). Of course, all of these terms could be listed as direct children of process and quality, but we think that a more detailed classification would benefit the users of the ontology and, especially, would make it easier to design other ontologies for vector-borne diseases. A similar consideration is valid for malaria prevention and vector control, terms that need to be included, and are now under the "place holder" process of malaria. It should be noted here that recently the Vector Surveillance and Management Ontology (VSMO) was published that covers the domain of vector control [22]. This development may make it easier to find a partial solution to the last mentioned problem.

\section{Conclusions}

It was not unexpected that IDOMAL had to undergo several updates, partial revisions and expansion during the three years after it was published, which all are summarized in this report. Not only is it legitimate to always try to obtain a better "product", but also some of the changes are dictated by the needs of the community (e.g. remedies and natural products) or recent developments in the field. As mentioned in the beginning, improved IT tools are becoming indispensable, especially as high throughput technology develops and provides more data. In the case of malaria and other vector borne diseases, this evolution is obvious. Only about ten years after the determination of the genome sequence of Anopheles gambiae [23] tens of genomes of different vectors have become available [24]. Although so far genes are usually only annotated with GO terms [25], the day is not far when they, and other data in genomic/biological databases, will also be annotated with ontological terms describing these domains, such as, for example, VectorBase, the database that covers arthropod disease vectors [26]. Moreover novel IT tools such as decision support systems are already making use of ontologies [27] and, even more, tools are planned that will be able to direct information to and from ontologies and data holders [28]. In the domain of vector-borne diseases, IDOMAL and MIRO, an ontology of Mosquito Insecticide Resistance [29] that has now been fully integrated in IDOMAL, are used by newly developed Decision Support Systems for vector-borne diseases [30,31]. Furthermore, VSMO also uses a several terms that have been imported from IDOMAL [22]. It becomes clear that the availability of all the new, open bio-medical ontologies provides ways to achieve enhanced interoperability between databases and to expand the title of the original publication of the Gene Ontology [32] to "tools for the unification of bio-medical sciences".

N.B. Both IDOMAL versions are available for downloading: the $\mathrm{OBO}$ version is at the OBO Foundry and at the NCBO BioPortal, while the OWL version is available at: http://anobase.vectorbase.org/idomal/.

\section{Abbreviations}

BFO: Basic formal ontology; FLU: Influenza ontology; GO: Gene ontology; IDO: Infectious disease ontology; IDODEN: Infectious disease ontologydengue; IDOMAL: Infectious disease ontology-malaria; MIRO: Mosquito insecticide resistance ontology; NCBO: National center for biomedical ontology; OGMS: Ontology for general medical science; OBO: Open and biomedical ontologies; OWL: Web ontology language; SYMP: Symptom ontology; VMSO: Vector surveillance and management ontology.

\section{Competing interests}

The authors declare that they have no competing interests.

\section{Authors' contributions}

PT was responsible for the final updating process and oversaw the regular operations; EM, VD and ED were responsible for individual parts of the update project; $\mathrm{CL}$ researched the domain of natural products and wrote the first draft of the paper (which all other authors helped finalize) and coordinated the study. All authors read and approved the final manuscript.

\section{Acknowledgements}

We would like to dedicate this paper to the memory of an outstanding malariologist, an excellent geneticist and, first of all, a very dear friend, Prof. Mario Coluzzi. We thank Dr. Frank Collins for his continuous support. This study was funded by the National Institutes of Health/National Institute for Allergy and Infectious Diseases (grant numbers HHSN266200400039C, HHSN272200900039C) in the frame of the VectorBase project and was also partially supported by grants 223736 (Transmalariabloc) and 201588

(Evimalar) of the FP7 (HEALTH) programme of the European Commission. CL was supported, in part, by an i-Move fellowship from the Polo d'Innovazione di Genetica Genomica e Biologia, Perugia.

\section{Author details}

${ }^{1}$ Institute of Molecular Biology and Biotechnology, Foundation for Research and Technology-Hellas, Heraklion, Crete, Greece. ${ }^{2}$ Institute of Molecular Biology and Biotechnology, Foundation for Research and Technology-Hellas and Department of Biology, University of Crete, Heraklion, Crete, Greece. ${ }^{3}$ Centre of Functional Genetics, Medical School, University of Perugia, Perugia, Italy.

Received: 29 May 2013 Accepted: 27 August 2013

Published: 13 September 2013 


\section{References}

1. World Health Organization: World Malaria Report 2012. http://www.who.int/ malaria/publications/world_malaria_report_2012/en/.

2. Mendis K, Rietveld A, Warsame M, Bosman A, Greenwood B, Wernsdorfer WH: From malaria control to eradication: the WHO perspective. Trop Med Intern Health 2009, 4:1-7.

3. World Health Organization: Global malaria control and elimination: report of a technical review 2008. http://whalibdoc.who.int/publications/2008/ 9789241596756_eng.pdf.

4. Topalis P, Dialynas E, Mitraka E, Deliyanni E, Siden-Kiamos I, Louis C: A set of ontologies to drive tools for the control of vector-borne diseases. J Biomed Inform 2011, 44:42-47.

5. Topalis P, Mitraka E, Bujila I, Deliyanni E, Dialynas E, Siden-Kiamos I, TroyeBlomberg M, Louis C: IDOMAL: an ontology for malaria. Malaria J 2010, 9(1):230

6. Cowell LG, Smith B: Infectious disease ontology. In Infectious disease informatics. Edited by Sintchenko V. New York: Springer; 2010:373-395.

7. Dengue Fever Ontology - Summary | NCBO BioPortal. http://bioportal. bioontology.org/ontologies/3174.

8. Dritsou V, Mitraka E, Topalis P, Louis C: Getting the best from two worlds: Converting between $\mathrm{OBO}$ and OWL formats. In OWL: Experiences and Directions Workshop: 27-28 May 2012. Edited by Klinov P, Horridge M. Heraklion: CEUR Workshop Proceedings. (ISSN: 1613-0073) http://ceur-ws. org/Nol-849/paper_1.pdf.

9. Grenon P: BFO in a Nutshell: A Bi-categorial Axiomatization of BFO and Comparison with DOLCE. IFOMIS Technical Report Series 06/2003. (ISSN 1611-4019), Medizinische Fakultät der Universität Leipzig, 2003.

10. Simon J, Dos Santos M, Fielding J, Smith B: Formal ontology for natural language processing and the integration of biomedical databases. Int J Med Inform 2006, 75:224-231.

11. Grenon P, Smith B, Goldberg L: Biodynamic ontology: applying BFO in the biomedical domain. Stud Health Technol Inform 2004, 102:20-38.

12. Garcia-Bustos JF, Gamo FJ: Antimalarial drug resistance and early drug discovery. Curr Pharm Des 2013, 19(2):270-281.

13. Fairhurst RM, Nayyar GM, Breman JG, Hallett R, Vennerstrom JL, Duong S, Ringwald P, Wellems TE, Plowe CV, Dondorp AM: Artemisinin-resistant malaria: research challenges, opportunities, and public health implications. Am J Trop Med Hyg 2012, 87(2):231-241.

14. Willcox M, Bodeker G, Rasoanaivo P: Traditional Medicinal Plants and Malaria. Boca Raton: CRC Press; 2004.

15. Whetzel PL, Noy NF, Shah NH, Alexander PR, Nyulas C, Tudorache T, Musen MA: BioPortal: enhanced functionality via new Web services from the National Center for Biomedical Ontology to access and use ontologies in software applications. Nucleic Acids Res 2011, 39(Web Server issue):541-545.

16. Scheuermann RH, Ceusters W, Smith B: Toward an Ontological Treatment of Disease and Diagnosis. In Proceedings of the 2009 AMIA Summit on Translational Bioinformatics, 15-17 March 2009. San Francisco: American Medical Informatics Association; 2009:116-120.

17. Influenza Ontology Wiki. http://influenzaontologywiki.igs.umaryland.edu/wiki/ index.php/Main_Page.

18. Symptomontologywiki. http://symptomontologywiki.igs.umaryland.edu/wiki/ index.php/Main_Page.

19. Goldfain A, Smith B, Cowell LG: Towards an ontological representation of resistance: the case of MRSA. J Biomed Inform 2011, 44(1):35-41.

20. Alphey L, Nimmo D, O'Connell S, Alphey N: Insect population suppression using engineered insects. Adv Exp Med Biol 2008, 627:93-103.

21. James AA: Gene drive systems in mosquitoes: rules of the road. Trends Parasitol 2005, 21(2):64-67.

22. Lozano-Fuentes S, Bandyopadhyay A, Cowell LG, Goldfain A, Eisen L: Ontology for vector surveillance and management. J Med Entomol 2013, 50(1):1-14.

23. Holt RA, Subramanian GM, Halpern A, Sutton GG, Charlab R, et al: The genome sequence of the malaria mosquito Anopheles gambiae. Science 2002, 298:129-149.

24. Home-Genome-NCBI. http://www.ncbi.nlm.nih.gov/genome/.

25. Gene Ontology Consortium: Gene Ontology annotations and resources. Nucleic Acids Res 2013, 41(Database issue):530-535.

26. Megy K, Emrich SJ, Lawson D, Campbell D, Dialynas E, et al: VectorBase: improvements to a bioinformatics resource for invertebrate vector genomics. Nucleic Acids Res 2012, 40(Database issue):729-734.
27. Haug PJ, Ferraro JP, Holmen J, Wu X, Mynam K, Ebert M, Dean N, Jones J: An ontology-driven, diagnostic modeling system. J Am Med Inform Assoc 2013: Epub ahead of print: PMID: 23523876.

28. Borrego L, Quaresma P: Processing medical reports to automatically populate ontologies. Stud Health Technol Inform 2013, 183:201-205.

29. Dialynas E, Topalis P, Vontas J, Louis C: MIRO and IRbase: IT tools for the epidemiological monitoring of insecticide resistance in mosquito disease vectors. PLoS Negl Tro Dis 2009, 3(6):e465. doi:10.1371/journal.pntd.0000465.

30. Lozano-Fuentes S, Barker CM, Coleman M, Coleman M, Park B, et al: Emerging Information Technologies to Provide Improved Decision Support for Surveillance, Prevention, and Control of Vector-Borne Diseases. In Efficient Decision Support Systems - Practice and Challenges in Biomedical Related Domain. Edited by Jao CS. ; 2011:89-114. InTech. ISBN 978-953-307-258-6.

31. Eisen L, Coleman M, Lozano-Fuentes S, McEachen N, et al: Multi-disease data management system platform for vector-borne diseases. PLOS Negl Tro Dis 2011, 5(3):e1016. doi:10.1371/journal.pntd.0001016.

32. Ashburner M, Ball CA, Blake JA, Botstein D, Butler H, et al: Gene ontology: tool for the unification of biology. The Gene Ontology Consortium. Nat Genet 2000, 25(1):25-29.

doi:10.1186/2041-1480-4-16

Cite this article as: Topalis et al:: IDOMAL: the malaria ontology revisited. Journal of Biomedical Semantics 2013 4:16.

\section{Submit your next manuscript to BioMed Central and take full advantage of:}

- Convenient online submission

- Thorough peer review

- No space constraints or color figure charges

- Immediate publication on acceptance

- Inclusion in PubMed, CAS, Scopus and Google Scholar

- Research which is freely available for redistribution 